\title{
ANALOGUE OF TRICOMI PROBLEM FOR CHARACTERISTICALLY LOADED HYPERBOLIC-PARABOLIC EQUATION WITH VARIABLE COEFFICIENTS
}

\author{
K.U. KHUBIEV
}

\begin{abstract}
In the work we study an analogue of Tricomi problems for characteristically loaded hyperbolic-parabolic equations with variable coefficients. We prove the unique solvability of the studied problem. The uniqueness of the solutions is proved by means of the maximum principle, while the existence is established by the method of integral equations.
\end{abstract}

Keywords: loaded equations, mixed equations, hyperbolic-parabolic equations, Tricomi problem, boundary value problem.

Mathematics Subject Classification: 35M12

\section{INTRODUCTION}

Mixed type equations have an important place in the theory of partial differential equations due to their theoretical and practical importance. One of the most important classes of partial differential equations are loaded mixed type equations. Monograph [1] by A.M. Nakhushev is devoted to studying local and nonlocal boundary value problems for loaded partial differential equations.

An analogue of Tricomi problem for a model hyperbolic-parabolic equation was studied first in work [2]. Local and nonlocal boundary value problems for hyperbolic-parabolic equations including the inverse problems and the problems for degenerate equations were studied by many authors (see, for instance, [3] $-[6]$ ).

At present, the theory of boundary value problems and inverse problems for hyperbolicparabolic equations including degenerate equations is being intensively developed. In this relation, we mention the following works. In [7] there was proved an apriori estimate for the classical solution to an analogue of Tricomi problem for a inhomogeneous model hyperbolic-parabolic equation with the right hand side in a Hölder class. In [8] for a mixed hyperbolic-parabolic equation in a rectangular domain, the inverse problem on determining an unknown right hand side was studied. A criterion for the uniqueness of the solution was established and the solution was constructed as the sum of a series over eigenfunctions of the corresponding one-dimensional spectral problem. In [9] there was studied a non-local interior problem with the Erdélyi-Kober operator for a model hyperbolic-parabolic equation. In [10], by the methods of spectral analysis, a criterion of the uniqueness of the solution was established for a problem for an equation in a rectangular domain degenerating in the hyperbolicity domain with a non-local condition relating the values of the sought solution corresponding to different types of the studied equation. The solution was constructed as a sum of the series over the eigenfunctions of the corresponding

K.U. Khubiev, Analogue of Tricomi problem for Characteristically loaded hyperbolicPARABOLIC EQUATION WITH VARIABLE COEFFICIENTS.

(c) Khubiev K.U. 2017.

Submitted July 7, 2016. 
one-dimensional spectral problem and the stability of the solution w.r.t. the non-local condition was established. In [11, [12] there were studied non-local boundary value problems with Bitsadze-Samarskii type condition for a second degenerating hyperbolic-parabolic equation. In [13], a boundary value problem with Dirichlet conditions on the characteristics in the parabolicity and hyperbolicity domains was studied for an inhomogeneous hyperbolic-parabolic equation with a characteristic line of type changing.

We also mention works on second and third order loaded hyperbolic-parabolic equations in various domains [14]-19]. In [14], boundary value problems for model second order loaded hyperbolic-parabolic equations were studied, when the type changing line is non-characteristic and for third order equations, when the type changing line is characteristic. In [15] there was proved the unique solvability of boundary value problems for a third order loaded equation with a hyperbolic and parabolic-hyperbolic operator. In [16], for hyperbolic-parabolic equation, the unique solvability of a non-local problem with generalized operator of fractional integrationdifferentiation in the boundary condition was studied. In [17], [18, the criterions for the uniqueness of the solution to the initial boundary value problems in a rectangular domain were established for various equations of mixed hyperbolic-parabolic type with loaded terms. The solutions were constructed as the sum of the series over eigenfunctions of the corresponding one-dimensional eigenvalue problem. In [19], there was studied an analogue of Tricomi problem for a model loaded hyperbolic-parabolic equation with a fractional derivative with a loading.

In the present work we consider the loaded hyperbolic-parabolic equation [1]

$$
\left\{\begin{array}{l}
u_{x x}-u_{y}+a_{1} u_{x}+c_{1} u+d_{1} u(x, 0)=f_{1}, \quad y>0 \\
u_{x x}-u_{y y}+a_{2} u_{x}+b_{2} u_{y}+c_{2} u+d_{2} u(x+y, 0)+e_{2} u(x-y, 0)=f_{2}, \quad y<0
\end{array}\right.
$$

in the domain $\Omega$ bounded by the segments $A A_{0}, B B_{0}, A_{0} B_{0}$ of the straight lines $x=0, x=l$, $y=h>0$, respectively, and by the characteristics $A C: x+y=0, B C: x-y=l$. By $\Omega_{1}$ and $\Omega_{2}$ we denote the parabolic and hyperbolic parts of the mixed domain $\Omega$, respectively, while by $J$ we denote the interval $0<x<l$ of the straight line $y=0, a_{i}=a_{i}(x, y), c_{i}=c_{i}(x, y)$, $d_{i}=d_{i}(x, y), f_{i}=f_{i}(x, y), b_{2}=b_{2}(x, y), e_{2}=e_{2}(x, y)$ are given functions in the class $C\left(\bar{\Omega}_{i}\right)$, $i=1,2$.

A regular in the domain $\Omega$ solution to the equation $(1.1)$ is a function $u(x, y)$ in the class $C(\bar{\Omega}) \cap C^{1}(\Omega) \cap C^{2}\left(\Omega_{2}\right) \cap C_{x}^{2}\left(\Omega_{1}\right), u_{x}, u_{y} \in L(J)$ satisfying equation $\sqrt{1.1}$ in $\Omega_{1} \cup \Omega_{2}$.

Problem T. Find a regular in the domain $\Omega$ solution $u(x, y)$ to equation (1.1) satisfying the boundary conditions

$$
\begin{aligned}
& u(0, y)=\varphi_{0}(y), \quad u(l, y)=\varphi_{l}(y), \quad 0 \leqslant y \leqslant h \\
& u(x / 2,-x / 2)=\psi(x), \quad 0 \leqslant x \leqslant l
\end{aligned}
$$

where $\varphi_{0}(y), \varphi_{l}(y), \psi(x)$ are given functions $\left.\varphi_{0}(y), \varphi_{l}(y) \in C[0, h], \psi(x) \in C[0, l] \cap C^{2}\right] 0, l[$, and $\varphi_{0}(0)=\psi(0)$.

In works [20], [21], for Problem T with $e_{2} \equiv 0$, there was proved the unique solvability of the studied problem under very strict conditions for the function $d_{2}$, namely, in the case, when the function $d_{2}$ depended in a certain way of the functions $a_{2}, b_{2}, c_{2}$. In this work these conditions are weakened essentially and as $d_{1}=d_{2}=e_{2} \equiv 0$, the obtained results coincides with the results provided in [3].

\section{UNIQUE SOLVABILITY THEOREM}

For Problem T we have the following theorem.

Theorem 2.1. Assume that 
1) in $\bar{\Omega}_{1}$ the functions $a_{1}(x, y), c_{1}(x, y), d_{1}(x, y), f_{1}(x, y)$ are continuous and satisfy Hölder condition in $x, a_{1}(x, 0) \in C^{1}[0, l]$, moreover,

$$
c_{1}(x, y)+d_{1}(x, y)<0, \quad d_{1}(x, y) \geqslant 0
$$

2) we have $a_{2}(x, y), b_{2}(x, y) \in C^{1}\left(\bar{\Omega}_{2}\right), c_{2}(x, y), d_{2}(x, y), e_{2}(x, y), f_{2}(x, y) \in C\left(\bar{\Omega}_{2}\right)$, and the function $u(x, y)$ possesses the property $\left(\frac{\partial}{\partial y}-\frac{\partial}{\partial x}\right) u \in C\left(\bar{\Omega}_{2} \backslash \bar{J}\right)$, moreover, the conditions

$$
\begin{aligned}
& a_{2}^{2}-b_{2}^{2}+2 a_{2 x}+2 b_{2 x}+2 a_{2 y}+2 b_{2 y}-4 c_{2} \geqslant 0, \\
& a_{2}(x, y)+b_{2}(x, y)>0, c_{2}(x, y)+d_{2}(x, y)+e_{2}(x, y) \geqslant 0, d_{2}(x, y) \leqslant 0, e_{2}(x, y) \leqslant 0
\end{aligned}
$$

hold true.

Then Problem $T$ is uniquely solvable.

Proof. Assume that there exists a solution $u(x, y)$ to Problem T. We denote

$$
u(x, 0)=\tau(x), \quad u_{y}(x, 0)=\nu(x) .
$$

Then it follows from the conditions of the problem that $\tau(0)=\varphi_{0}(0)=\psi(0), \tau(l)=\varphi_{l}(0)$, $\tau(x) \in C(\bar{J}) \cap C^{1}(J), \nu(x) \in C(J) \cap L(J)$.

2.1. Uniqueness of the solution to Problem T. We consider the homogeneous Problem $\mathrm{T}$, that is, $\varphi_{0}(y)=\varphi_{l}(y)=\psi(x) \equiv 0, f_{1}(x, y)=f_{2}(x, y) \equiv 0$. In $\Omega_{2}$, equation (1.1) in characteristic coordinates $\xi=x+y, \eta=x-y$ becomes

$$
v_{\xi \eta}+p v_{\xi}+q v_{\eta}+r v+\lambda v(\xi, \xi)+\mu v(\eta, \eta)=0,
$$

where $4 p=a_{2}+b_{2}, 4 q=a_{2}-b_{2}, 4 r=c_{2}, 4 \lambda=d_{2}, 4 \mu=e_{2}, v=v(\xi, \eta)=u(x, y)$, and $\Omega^{-} \cup A B$ become the domain $D=\{(\xi, \eta): 0<\xi<\eta<l\}$.

Following [22], let us show that a positive maximum of the function $v(\xi, \eta)$ in $\bar{D}$ can be attained only in the segment $0 \leqslant \xi=\eta \leqslant l$. Indeed, let $(\varepsilon, \delta)$ is arbitrary fixed point in the domain $D, \varepsilon, \delta=$ const $>0$. As it follows from the assumptions of Theorem 2.1, the function $p$ in the domain $D$ has a continuous derivative w.r.t. $\xi$, and $q$ is continuous in $D$. In the class of the functions $v=v(\xi, \eta)$ having in $D$ first and second mixed derivatives, equation (2.5) is equivalent to the equation

$$
\left(q_{1} v_{\eta}+p_{1} v\right)_{\xi}+r_{1} v+\lambda_{1} v(\xi, \xi)+\mu_{1} v(\eta, \eta)=0
$$

or to the loaded first order equation

$$
\begin{aligned}
& q_{1}(\xi, \eta) v_{\eta}(\xi, \eta)+p_{1}(\xi, \eta) v(\xi, \eta)+\int_{\varepsilon}^{\xi} r_{1}\left(\xi_{1}, \eta\right) v\left(\xi_{1}, \eta\right) d \xi_{1} \\
& =q_{1}(\varepsilon, \eta) v_{\eta}(\varepsilon, \eta)+p_{1}(\varepsilon, \eta) v(\varepsilon, \eta)-\int_{\varepsilon}^{\xi} \lambda_{1}\left(\xi_{1}, \eta\right) v\left(\xi_{1}, \xi_{1}\right) d \xi_{1}-\int_{\varepsilon}^{\xi} \mu_{1}\left(\xi_{1}, \eta\right) v(\eta, \eta) d \xi_{1},
\end{aligned}
$$

where

$$
\begin{aligned}
& r_{1}=r q_{1}-p_{1 \xi}, \quad p_{1}=p q_{1}, \quad q_{1}=\exp \int_{\delta}^{\xi} q(t, \eta) d t \\
& \lambda_{1}=\lambda q_{1}, \quad \mu_{1}=\mu q_{1}, \quad 0<\xi<\eta<l .
\end{aligned}
$$


We rewrite (2.6) as

$$
\begin{aligned}
q_{1}(\xi, \eta) v_{\eta}(\xi, \eta)= & \int_{\varepsilon}^{\xi}\left[v(\xi, \eta)-v\left(\xi_{1}, \eta\right)\right] r_{1}\left(\xi_{1}, \eta\right) d \xi_{1}+q_{1}(\varepsilon, \eta)\left[v_{\eta}(\varepsilon, \eta)+p(\varepsilon, \eta) v(\varepsilon, \eta)\right] \\
& +\int_{\varepsilon}^{\xi}\left[v(\xi, \eta)-v\left(\xi_{1}, \xi_{1}\right)\right] \lambda_{1}\left(\xi_{1}, \eta\right) d \xi_{1}+\int_{\varepsilon}^{\xi}[v(\xi, \eta)-v(\eta, \eta)] \mu_{1}\left(\xi_{1}, \eta\right) d \xi_{1} \\
& -v(\xi, \eta)\left[p_{1}(\xi, \eta)+\int_{\varepsilon}^{\xi}\left[r\left(\xi_{1}, \eta\right) q_{1}\left(\xi_{1}, \eta\right)+\lambda_{1}\left(\xi_{1}, \eta\right)+\mu_{1}\left(\xi_{1}, \eta\right)\right] d \xi_{1}\right] \\
= & \int_{\varepsilon}^{\xi}\left[v(\xi, \eta)-v\left(\xi_{1}, \eta\right)\right] r_{1}\left(\xi_{1}, \eta\right) d \xi_{1}+q_{1}(\varepsilon, \eta)\left[v_{\eta}(\varepsilon, \eta)+p(\varepsilon, \eta) v(\varepsilon, \eta)\right] \\
& +\int_{\varepsilon}^{\xi}\left[v(\xi, \eta)-v\left(\xi_{1}, \xi_{1}\right)\right] \lambda_{1}\left(\xi_{1}, \eta\right) d \xi_{1}+\int_{\varepsilon}^{\xi}[v(\xi, \eta)-v(\eta, \eta)] \mu_{1}\left(\xi_{1}, \eta\right) d \xi_{1} \\
& -v(\xi, \eta)\left[p_{1}(\varepsilon, \eta)+\int_{\varepsilon}^{\xi}\left[r\left(\xi_{1}, \eta\right)+\lambda\left(\xi_{1}, \eta\right)+\mu\left(\xi_{1}, \eta\right)\right] q_{1}\left(\xi_{1}, \eta\right) d \xi_{1}\right] .
\end{aligned}
$$

Suppose that a positive maximum of the function $v(\xi, \eta)$ being a regular solution to equation (2.5) is attained in $\bar{D}$ at a point $\left(\xi_{0}, \eta_{0}\right), 0<\xi_{0}<\eta_{0} \leqslant l$. By 2.7$)$ as $\xi=\xi_{0}, \eta=\eta_{0}, \varepsilon \rightarrow 0$, we have

$$
\begin{aligned}
q_{1}\left(\xi_{0}, \eta_{0}\right) & v_{\eta}\left(\xi_{0}, \eta_{0}\right)=\int_{0}^{\xi_{0}}\left[v\left(\xi_{0}, \eta_{0}\right)-v\left(\xi_{1}, \eta_{0}\right)\right] r_{1}\left(\xi_{1}, \eta_{0}\right) d \xi_{1} \\
& +\int_{0}^{\xi_{0}}\left[v\left(\xi_{0}, \eta_{0}\right)-v\left(\xi_{1}, \xi_{1}\right)\right] \lambda_{1}\left(\xi_{1}, \eta_{0}\right) d \xi_{1} \\
& +\int_{0}^{\xi_{0}}\left[v\left(\xi_{0}, \eta_{0}\right)-v\left(\eta_{0}, \eta_{0}\right)\right] \mu_{1}\left(\xi_{1}, \eta_{0}\right) d \xi_{1} \\
& -v\left(\xi_{0}, \eta_{0}\right)\left[p_{1}\left(\xi_{0}, \eta_{0}\right)+\int_{0}^{\xi}\left[r\left(\xi_{1}, \eta_{0}\right)+\lambda\left(\xi_{1}, \eta_{0}\right)+\mu\left(\xi_{1}, \eta_{0}\right)\right] q_{1}\left(\xi_{1}, \eta_{0}\right) d \xi_{1}\right] \\
& +q_{1}(0, \eta)\left[v_{\eta}(0, \eta)+p(0, \eta) v(0, \eta)\right] .
\end{aligned}
$$

It follows from the assumptions of Theorem 2.1 that $p, p_{\xi}, q, r, \lambda$ and $\mu$ belong to $C(0 \leqslant \xi<$ $\eta \leqslant l), v_{\eta} \in C(0 \leqslant \xi<\eta \leqslant l)$. Moreover, by (2.2), (2.3) we obtain that

$$
\begin{gathered}
r_{1}(\xi, \eta) \leqslant 0, \quad \lambda(\xi, \eta) \leqslant 0, \quad \mu(\xi, \eta) \leqslant 0, \\
p_{1}(\xi, \eta)+\int_{0}^{\xi}\left[r\left(\xi_{1}, \eta\right)+\lambda\left(\xi_{1}, \eta\right)+\mu\left(\xi_{1}, \eta\right)\right] q_{1}\left(\xi_{1}, \eta\right) d \xi_{1}>0,
\end{gathered}
$$


while it follows from $\psi(x) \equiv 0$ that

$$
v_{\eta}(0, \eta)+p(0, \eta) v(0, \eta)=0 .
$$

Thus, taking into consideration the assumptions of Theorem 2.1 and that $q_{1}(\xi, \eta)>0$, by 2.8) we obtain that $v_{\eta}\left(\xi_{0}, \eta_{0}\right)<0$. But this contradicts the made assumption since at the point $\left(\xi_{0}, \eta_{0}\right)$ of the positive maximum we have $v_{\eta}\left(\xi_{0}, \eta_{0}\right) \geqslant 0$. Therefore, a positive maximum of the function $v(\xi, \eta)$ in $\bar{D}$ is attained only in the segment $0 \leqslant \xi=\eta \leqslant l$ and under the assumptions of Theorem 2.1, the solution $u(x, y)$ to equation (1.1) as $y<0$, attains its positive maximum in $\bar{\Omega}_{2}$ in an interior point $\left(x_{0}, 0\right)$ of the segment $A \bar{B}$ and at the point of the positive maximum we have

$$
\nu\left(x_{0}\right) \geqslant 0 .
$$

Remark 2.1. We observe that as $d_{2}=e_{2} \equiv 0$, the obtained extremum principle for a loaded hyperbolic equation coincides with Agmon-Nirenberg-Protter principle formulated for the hyperbolic equation in [22] and the obtained conditions are in agreement with the conditions obtained in work 23]. A short survey of the results on the maximum principle for the mixed type equation was given in work [24].

Similar to [22], let us show that as $y>0$, the positive maximum of the function $u(x, y)$ in $\bar{\Omega}^{+}$can be attained only in $A A_{0}, A B, B B_{0}$. Assume that a regular solution $u(x, y)$ to equation (1.1) as $y>0$ attains its positive maximum at a point $\left(x_{0}, y_{0}\right) \in \Omega^{+}$. The necessary maximum condition for the function $u$ at the point $\left(x_{0}, y_{0}\right)$ is of the form: $u_{x}=0, u_{y}=0, u_{x x} \leqslant 0$. Taking this into consideration, by (1.1) we find

$$
c_{1}\left(x_{0}, y_{0}\right) u\left(x_{0}, y_{0}\right)+d_{1}\left(x_{0}, y_{0}\right) u\left(x_{0}, 0\right)=-u_{x x}\left(x_{0}, y_{0}\right) \geqslant 0 .
$$

On the other hand, under assumptions (2.1) of Theorem 2.1, $c_{1}+d_{1}<0, d_{1} \geqslant 0$, we obtain:

$$
\begin{aligned}
c_{1}\left(x_{0}, y_{0}\right) & u\left(x_{0}, y_{0}\right)+d_{1}\left(x_{0}, y_{0}\right) u\left(x_{0}, 0\right) \\
= & c_{1}\left(x_{0}, y_{0}\right) u\left(x_{0}, y_{0}\right)+d_{1}\left(x_{0}, y_{0}\right) u\left(x_{0}, 0\right)+d_{1}\left(x_{0}, y_{0}\right) u\left(x_{0}, y_{0}\right)-d_{1}\left(x_{0}, y_{0}\right) u\left(x_{0}, y_{0}\right) \\
= & {\left[c_{1}\left(x_{0}, y_{0}\right)+d_{1}\left(x_{0}, y_{0}\right)\right] u\left(x_{0}, y_{0}\right)-d_{1}\left(x_{0}, y_{0}\right)\left[u\left(x_{0}, y_{0}\right)-u\left(x_{0}, 0\right)\right]<0 . }
\end{aligned}
$$

The obtained contradiction is because of the wrong assumption and $\left(x_{0}, y_{0}\right) \notin \Omega^{+}$. The statement that the maximum point does not belong to $A_{0} B_{0}$ can be proved in the same way as in the case $y_{0}<h$ with the only difference that the necessary extremum condition $u_{y}\left(x_{0}, y_{0}\right)=0$ as $y_{0}<h$ is replaced by the condition $u_{y}\left(x_{0}, y_{0}\right) \geqslant 0$ as $y_{0}=h$.

Thus, under the assumptions of Theorem 2.1 we have that the positive maximum of the function $u(x, y)$ can be attained only in the segments $A A_{0}, B B_{0}, A B$.

Let us show that for the function $u(x, y)$, each interior point $\left(x_{0}, 0\right)$ in the segment $A B$ can not be a point of a positive maximum. Indeed, by the continuity of the derivatives $u_{x}, u_{y}, u_{x x}$ in equation (1.1), we can pass to the limit as $y \rightarrow+0$ to obtain

$$
\tau^{\prime \prime}(x)+a_{1}(x, 0) \tau^{\prime}(x)+\left[c_{1}(x, 0)+d_{1}(x, 0)\right] \tau(x)-\nu(x)=0 .
$$

By conditions (2.1) of Theorem 2.1 and by 2.10), at the point of a positive maximum we have $\nu\left(x_{0}\right)<0$ that contradicts inequality (2.9). This yields that the function $u(x, y)$ can not attain a positive maximum in the interior points $\left(x_{0}, 0\right)$ of the segment $A B$. Hence, under the assumptions of Theorem 2.1, the positive maximum of the function $u(x, y)$ can be attained only in the segments $A A_{0}$ and $B B_{0}$. Since $\varphi_{0}(y)=\varphi_{l}(y) \equiv 0$, we conclude that the maximum of the function $u(x, y)$ in $\bar{\Omega}$ is equal to zero. In the same way one can prove that the function $u(x, y)$ can not have a negative minimum and the minimum of the function $u(x, y)$ is zero. Therefore, the homogeneous problem corresponding to Problem T has only the trivial solution $u(x, y) \equiv 0$ that implies the uniqueness of a solution to Problem T. 
2.2. Existence of solution to Problem T. Solving Cauchy problem [25] for equation (1.1) in the domain $\Omega_{2}$ as for the inhomogeneous wave equation with the right hand side $f_{2}(x, y)-d_{2}(x, y) \tau(x+y)-e_{2}(x, y) \tau(x-y)$, we obtain

$$
\begin{aligned}
u(x, y)= & \frac{1}{2}[R(x, y ; x+y, 0) \tau(x+y)+R(x, y ; x-y, 0) \tau(x-y)] \\
& -\frac{1}{2} \int_{x-y}^{x+y}\left[R_{\eta}(x, y ; \xi, 0)+b_{2}(\xi, 0) R(x, y ; \xi, 0)\right] \tau(\xi) d \xi+\frac{1}{2} \int_{x-y}^{x+y} R(x, y ; \xi, 0) \nu(\xi) d \xi \\
& +\frac{1}{2} \int_{y}^{0} \int_{x+y-\eta}^{x-y+\eta} R(x, y ; \xi, \eta)\left[f_{2}(\xi, \eta)-d_{2}(\xi, \eta) \tau(\xi+\eta)-e_{2}(\xi, \eta) \tau(\xi-\eta)\right] d \xi d \eta,
\end{aligned}
$$

where $R(x, y ; \xi, \eta)$ is the Riemann function introduced as the solution to the Goursat problem

$$
\begin{aligned}
& R_{1}=\left.R(x, y ; \xi, \eta)\right|_{\eta=x+y-\xi}=\exp \left(\frac{1}{2} \int_{x}^{\xi}\left[a_{2}(t, x+y-t)+b_{2}(t, x+y-t)\right] d t\right) \\
& R_{2}=\left.R(x, y ; \xi, \eta)\right|_{\eta=\xi-x+y}=\exp \left(\frac{1}{2} \int_{x}^{\xi}\left[a_{2}(t, t-x+y)+b_{2}(t, t-x+y)\right] d t\right), \\
& R(x, y ; x, y)=1
\end{aligned}
$$

for the equation

$$
R_{\xi \xi}-R_{\eta \eta}-\left(a_{2} R\right)_{\xi}-\left(b_{2} R\right)_{\eta}+c_{2} R=0 .
$$

Satisfying condition (1.3) for (2.11), we obtain

$$
\begin{aligned}
& R_{2}(x / 2,-x / 2 ; x, 0) \tau(x)+\int_{0}^{x}\left[R_{\eta}(x / 2,-x / 2 ; \xi, 0)+b_{2}(\xi, 0) R(x / 2,-x / 2 ; \xi, 0)\right] \tau(\xi) d \xi \\
& -\int_{-x / 2}^{0} \int_{-\eta}^{x+\eta} d_{2}(\xi, \eta) R(x / 2,-x / 2 ; \xi, \eta) \tau(\xi+\eta)-e_{2}(\xi, \eta) R(x / 2,-x / 2 ; \xi, \eta) \tau(\xi-\eta) d \xi d \eta \\
& =2 \psi(x)-R_{1}(x / 2,-x / 2 ; 0,0) \psi(0)+\int_{0}^{-x / 2} \int_{x+\eta}^{-\eta} R(x / 2,-x / 2 ; \xi, \eta) f_{2}(\xi, \eta) d \xi d \eta \\
& +\int_{0}^{x} R(x / 2,-x / 2 ; \xi, 0) \nu(\xi) d \xi .
\end{aligned}
$$

Switching the integration limits in the double integrals, we obtain

$$
\begin{aligned}
& \int_{-x / 2}^{0} \int_{-\eta}^{x+\eta} d_{2}(\xi, \eta) R(x / 2,-x / 2 ; \xi, \eta) \tau(\xi+\eta) d \xi d \eta \\
= & \int_{0}^{x} \tau(\xi) \int_{\frac{\xi-x}{2}}^{0} d_{2}(\xi-\eta, \eta) R(x / 2,-x / 2 ; \xi-\eta, \eta) d \eta d \xi
\end{aligned}
$$




$$
\begin{aligned}
& \int_{-x / 2}^{0} \int_{-\eta}^{x+\eta} e_{2}(\xi, \eta) R(x / 2,-x / 2 ; \xi, \eta) \tau(\xi-\eta) d \xi d \eta \\
& =\int_{0}^{x} \tau(\xi) \int_{-\xi / 2}^{0} e_{2}(\xi+\eta, \eta) R(x / 2,-x / 2 ; \xi+\eta, \eta) d \eta d \xi
\end{aligned}
$$

and denoting

$$
\begin{aligned}
& K_{1}(x, \xi)=\int_{\frac{\xi-x}{2}}^{0} d_{2}(\xi-\eta, \eta) R(x / 2,-x / 2 ; \xi-\eta, \eta) d \eta+\int_{-\xi / 2}^{0} e_{2}(\xi+\eta, \eta) R(x / 2,-x / 2 ; \xi+\eta, \eta) d \eta, \\
& K(x, \xi)=\frac{K_{1}(x, \xi)-R_{\eta}(x / 2,-x / 2 ; \xi, 0)-b_{2}(\xi, 0) R(x / 2,-x / 2 ; \xi, 0)}{R_{2}(x / 2,-x / 2 ; x, 0)}, \\
& g_{1}(x)=\frac{2 \psi(x)-R_{1}(x / 2,-x / 2 ; 0,0) \psi(0)+\int_{0}^{-x / 2} \int_{x+\eta}^{-\eta} R(x / 2,-x / 2 ; \xi, \eta) f_{2}(\xi, \eta) d \xi d \eta}{R_{2}(x / 2,-x / 2 ; x, 0)}, \\
& g_{2}(x)=\int_{0}^{x} \frac{R(x / 2,-x / 2 ;, \xi, 0) \nu(\xi)}{R_{2}(x / 2,-x / 2 ; x, 0)} d \xi, \quad g(x)=g_{1}(x)+g_{2}(x),
\end{aligned}
$$

we obtain the second kind Volterra integral equation

$$
\tau(x)-\int_{0}^{x} K(x, \xi) \tau(\xi) d \xi=g(x) .
$$

Resolving the obtained integral equation w.r.t. $\tau(x)$, we get the first functional relation for $\tau(x)$ and $\nu(x)$ generated by the domain $\Omega_{2}$ in the form

$$
\tau(x)-\int_{0}^{x} T(x, \xi) \nu(\xi) d \xi=\rho(x),
$$

where

$$
\begin{aligned}
& T(x, \xi)=\frac{R(x / 2,-x / 2, \xi, 0)+\int_{\xi}^{x} \Gamma(x, t) R(t / 2,-t / 2 ; \xi, 0) d t}{R_{2}(x / 2,-x / 2 ; x, 0)}, \\
& \rho(x)=g_{1}(x)+\int_{0}^{x} \Gamma(x, \xi) g_{1}(\xi) d \xi,
\end{aligned}
$$

and $\Gamma(x, \xi)$ is the resolvent of the kernel $K(x, \xi)$.

Remark 2.2. We observe that as $d_{2}=e_{2}=f_{2} \equiv 0$, similar relations for equation (1.1) in $\Omega_{2}$ were obtained in [3], [26].

For inhomogeneous equation (1.1), relation 2.10) casts into the form

$$
\tau^{\prime \prime}(x)+a_{1}(x, 0) \tau^{\prime}(x)+\left[c_{1}(x, 0)+d_{1}(x, 0)\right] \tau(x)=f_{1}(x, 0)+\nu(x) .
$$

This is why the second functional relation for the functions $\tau(x)$ and $\nu(x)$ generated by the domain $\Omega_{1}$ is defined as the solution to equation (2.15) obeying the boundary conditions

$$
\tau(0)=\varphi_{0}(0), \quad \tau(l)=\varphi_{l}(0) .
$$


Under the assumptions of Theorem 2.1 on the smoothness of the functions $a_{1}, c_{1}, d_{1}, f_{1}$, it is of the form

$$
\tau(x)=f(x)-\int_{0}^{l} G(x, \xi) \nu(\xi) d \xi
$$

where

$$
\begin{aligned}
f(x)= & \varphi_{0}(0)+x\left\{\varphi_{l}(0)-\varphi_{0}(0)\right\}+\int_{0}^{l}\left[G ( x , \xi ) \left(f_{1}(\xi, 0)\right.\right. \\
& \left.\left.-\left\{a_{1}(\xi, 0)+\xi\left[c_{1}(\xi, 0)+d_{1}(\xi, 0)\right]\right\}\left\{\varphi_{l}(0)-\varphi_{0}(0)\right\}-\varphi_{0}(0)\left[c_{1}(\xi, 0)+d_{1}(\xi, 0)\right]\right)\right] d \xi,
\end{aligned}
$$

and $G(x, \xi)$ is the Green function with the properties [25]

a) in the segments $0 \leqslant x<\xi, \xi<x \leqslant l$, this function and its derivatives up to the second order are continuous and the function solves the equation adjoint for equation 2.15); moreover, for each fixed $\xi, 0<\xi<l$, as a function of $x$, it satisfies homogeneous boundary conditions (2.16);

b) as a function of $\xi$, the function is continuous at the point $\xi=x$, while its first derivative w.r.t. $x$ has a jump and $G_{x}(x, x+0)-G_{x}(x, x-0)=1$.

Excluding $\tau(x)$ from (2.14) and (2.17), we obtain the integral equation

$$
\int_{0}^{x} T(x, \xi) \nu(\xi) d \xi+\rho(x)=f(x)-\int_{0}^{l} G(x, \xi) \nu(\xi) d \xi .
$$

Differentiating it w.r.t. $x$ and taking into consideration that $T(x, x) \equiv 1$, we have

$$
\nu(x)-\int_{0}^{x} T_{1}(x, \xi) \nu(\xi) d \xi=f^{\prime}(x)-\rho^{\prime}(x)-\int_{0}^{l} G_{x}(x, \xi) \nu(\xi) d \xi,
$$

where $T_{1}(x, \xi)=\frac{T_{x}(x, \xi)}{R_{2}(x / 2,-x / 2, x, 0)}$. Solving equation 2.18 , we obtain

$$
\nu(x)+\int_{0}^{l} K_{2}(x, t) \nu(t) d t=\sigma(x) .
$$

Here

$$
\begin{aligned}
& K_{2}(x, t)=G_{x}(x, t)+\int_{0}^{x} \Gamma_{1}(x, \xi) G_{x}(\xi, t) d \xi \\
& \sigma(x)=f^{\prime}(x)-\rho^{\prime}(x)+\int_{0}^{x} \Gamma_{1}(x, \xi)\left[f^{\prime}(\xi)-\rho^{\prime}(\xi)\right] d \xi,
\end{aligned}
$$

and $\Gamma_{1}(x, \xi)$ is the resolvent of the kernel $T_{1}(x, \xi)$.

Since we seek $\nu(x)$ in the class of continuous functions integrable in an interval, the function $\sigma(x)$ should be also in the same class. By the properties of the functions $G(x, \xi)$ and $R(x, y, \xi, \eta)$ we conclude that the function $K_{2}(x, \xi)$ is continuous and continuously differentiable in $x$ in the segment $0 \leqslant x<\xi, \xi<x \leqslant l$, while the function $\sigma(x)$ is continuously differentiable in $0<x<l$.

The solvability of second kind Fredholm equation $(2.19)$ in the class of continuous functions integrable on the interval is implied by the uniqueness of the solution to Problem $\mathrm{T}$. 
In view of (2.19), the conditions imposed for the coefficients of equation (2.15), the aforementioned properties of the Green function and the identity

$$
\frac{d^{2} G(x, \xi)}{d x^{2}}=\frac{d}{d x}\left[a_{1}(x, 0) G(x, \xi)\right]-\left[c_{1}(x, 0)+d_{1}(x, 0)\right] G(x, \xi)
$$

valid as $x \neq \xi$, we have

$$
\begin{aligned}
\nu^{\prime}(x)= & \sigma^{\prime}(x)+\nu(x)-\int_{0}^{l}\left(\frac{d}{d x}\left[a_{1}(x, 0) G(x, t)\right]-\left[c_{1}(x, 0)+d_{1}(x, 0)\right] G(x, t)\right) \nu(t) d t \\
& -\int_{0}^{l} \Gamma_{1}(x, x) G_{x}(x, t) \nu(t) d t-\int_{0}^{l} \int_{0}^{x} \Gamma_{1 x}(x, \xi) G_{x}(\xi, t) \nu(t) d \xi d t,
\end{aligned}
$$

which implies easily that $\nu(x) \in C^{1}(J)$.

Once $\nu(x)$ is found, the function $\tau(x)$ is found by 2.14) or 2.17) and $\tau(x) \in C(\bar{J}) \cap C^{2}(J)$. Then solving Problem $\mathrm{T}$ in the domain $\Omega$ is reduced to solving the Cauchy problem for equation (1.1) in $\Omega_{2}$, that is, it is determined by formula (2.11), while in $\Omega_{1}$ it is reduced to solving Dirichlet problem for equation (1.1) since the functions $a_{1}(x, y), c_{1}(x, y), d_{1}(x, y), f_{1}(x, y)$ are continuous and satisfy Hölder condition in $x$ [27. The proof of Theorem 2.1 is complete.

Remark 2.3. We note that as in [3], condition (2.2) of Theorem 2.1 fails as $\left|a_{2}\right|=\left|b_{2}\right| \equiv$ const if $c_{2} \neq 0$ and this is why this case should be considered independently.

\section{BIBLIOGRAPHY}

1. A.M. Nakhushev. Loaded equations and their applications. Nauka, Moscow (2012). (in Russian).

2. L.A. Zolina. On a boundary value problem for a model equation of hyperbolo-parabolic type // Zhurn. Vychisl. Matem. Matem. Fiz. 6:6, 991-1001 (1966). (in Russian).

3. T.D. Dzhuraev, A.S. Sopuev, M. Mamazhanov. Boundary value problems for parabolic-hyperbolic equations. FAN, Tashkent (1986). (in Russian).

4. H.G. Bzkikhatlov, A.M. Nakhushev. A boundary value problem for a mixed equation of parabolichyperbolic type // Dokl. Akad. Nauk SSSR. 183:2, 261-264 (1968). [Sov. Math. Dokl. 9, 1349-1352 (1968).]

5. A.M. Nakhushev, Problems with shifts for partial differential equations. Nauka, Moscow (2006). (in Russian).

6. V.A. Eleev. Some boundary-value problems with translations for a parabolic - hyperbolic mixed equation // Differ. Uravn. 14:1, 22-29 (1978). [Differ. Equats. 14:1, 15-21 (1978).]

7. E.I. Moiseev, N.Yu. Kapustin. Sharpening an a priori estimate of the solution of a well-known problem for a parabolic-hyperbolic equation // Dokl. RAN. 427:5, 591-592 (2009). [Dokl. Math. 80:1, 563-564 (2009).]

8. K.B. Sabitov, E.M. Safin. The inverse problem for an equation of mixed parabolic-hyperbolic type // Matem. Zametki. 87:6, 907-918 (2010). [Math. Notes. 87:6, 880-889 (2010).]

9. Z.A. Nakhusheva. Nonlocal problem for the Lavrent'ev-Bitsadze equation and its analogs in the theory of equations of mixed parabolic-hyperbolic type // Differ. Uravn. 49:10, 1332-1339 (2013). [Differ. Equat. 49:10, 1299-1306 (2013).]

10. K.B. Sabitov, S.N. Sidorov. On a nonlocal problem for a degenerating parabolic-hyperbolic equation // Differ. Uravn. 50:3, 356-365 (2014). [Differ. Equat. 50:3, 352-361 (2014).]

11. N.B. Islamov. Analogue of Bitsadze-Samarskii problem for a class of parabolic-hyperbolic equations of second kind // Ufimskij Matem. Zhurn. 7:1, 31-45 (2015). [Ufa Math. J. 7:1, 31-45 (2015).]

12. M.S. Salakhitdinov, N.B. Islamov. Nonlocal boundary-value problem with BitsadzeSamarskii condition for equation of parabolic-hyperbolic type of the second kind // Izv. VUZov. Matem. 6, 43-52 (2015). [Russ. Math. (Izvestiya VUZ. Matem.) 59:6, 34-42 (2015).]

13. D.A. Gulyaev. On an inhomogeneous problem for parabolic-hyperbolic equation // Differ. Uravn. 52:10, 1423-1425 (2016). [Differ. Equat. 52:10, 1371-1373 (2016).] 
14. V.A. Eleev. Some boundary value problems for mixed loaded equations of second and third order // Differ. Uravn. 30:2, 230-237 (1994). [Differ. Equat. 30:2, 210-217 (1994).]

15. I.I. Baltaeva, B. Islomov. Boundary value problems for the loaded third order equations of the hyperbolic and mixed types // Ufimskij Matem. Zhurn. 3:3, 15-25 (2011). [Ufa Math. J. 3:3, 15-25 (2011).]

16. A.V. Tarasenko. Solvability of a nonlocal problem for a loaded parabolic-hyperbolic equation // Izv. VUZov. Matem. 1, 73-81 (2013). [Russ. Math. (Izv. VUZ. Matem.) 57:1, 64-71 (2013).]

17. K.B. Sabitov. Initial boundary value problem for a loaded parabolic-hyperbolic equation // Dokl. Adygskoi (Cherkesskoi) Mezhdun. Akad. Nauk. 11:1, 66-73 (2009). (in Russian).

18. K.B. Sabitov. Initial-boundary problem for parabolic-hyperbolic equation with loaded summands // Izv. VUZov. Matem. 6, 31-42 (2015). [Russ. Math. (Izv. VUZ. Matem). 59:6, 23-33 (2015).]

19. K.U. Khubiev. Analogue of Tricomi problem for loaded hyperbolic-parabolic equation with a fractional derivative at load // Dokl. Adygskoi (Cherkesskoi) Mezhdun. Akad. Nauk. 17:3, 54-59 (2015). (in Russian).

20. K.U. Khubiev. Analogue of Tricomi problem for loaded mixed type equation with variable coeffcients // Dokl. Adygskoi (Cherkesskoi) Mezhdun. Akad. Nauk. 8:2, 69-72 (2006). (in Russian).

21. K.U. Khubiev. Analogue of Tricomi problem for loaded hyperbolic-parabolic equation with variable coefficients // Vestn. Samar. Gos. Tekhn. Univ. Ser. Fiz.-Mat. Nauki. 2(15), 155-158 (2007). (in Russian).

22. A.M. Nakhushev. Equations of mathematical biology. Vysshaya Schkola, Moscow (1995). (in Russian).

23. S. Agmon, L. Nirenberg, M. Protter. A maximum principle for a class of hyperbolic equations and applications to equations of mixed elliptic-hyperbolic type // Comm. Pure Appl. Math. 6:4, 455-470 (1953).

24. K.B. Sabitov. Maximum principle for an equation of mixed type // Differ. Uravn. 24:11, 1967-1976 (1988). [Differ. Equat. 24:11, 1322-1329 (1988).]

25. S.L. Sobolev. Equations of mathematical physics. Nauka, Moscow (1966). (in Russian).

26. S.P. Pul'kin. The Tricomi problem for the general equation of Lavrentiew-Bitzadze // Dokl. Akad. Nauk SSSR. 118:1, 38-41 (1958). (in Russian).

27. A.M. Il'in, A.S. Kalashnikov, O.A. Oleinik. Linear equations of the second order of parabolic type // Uspekhi Matem. Nauk. 17:3(105), 3-141 (1962). [Russ. Math. Surv. 17:3, 1-143 (1962).]

Kazbek Uzeirovich Khubiev,

Institute of Applied Mathematics and Automation,

Kabardino-Balkar Scientific Center, RAS,

Shortanova str. 89-a,

360000, Nalchik, Russia

E-mail: khubiev_math@mail.ru 